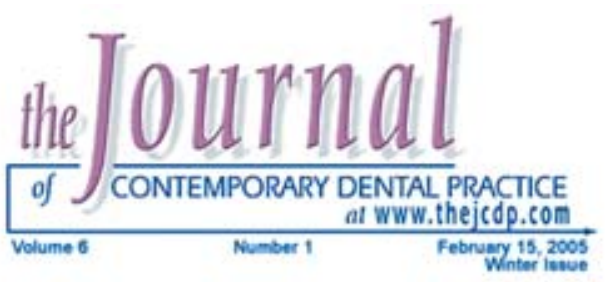

\title{
An Evaluation of Survival of Space Maintainers: A Six-year Follow-up Study
}

\author{
Ozlem Tulunoglu, DDS, PhD; Tezer Ulusu, DSD, PhD;
}

Yasemin Genç, MS, PhD

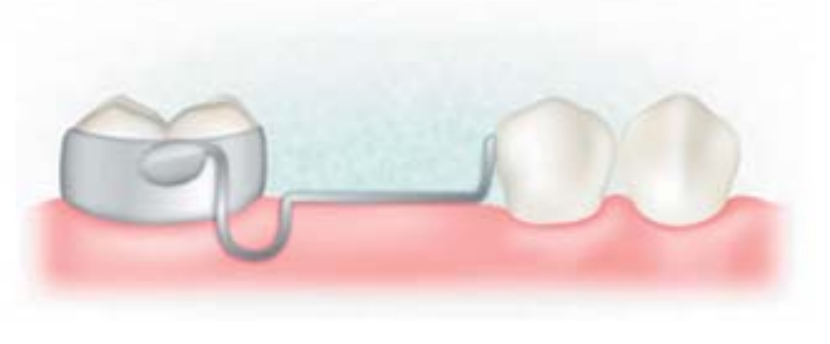

\begin{abstract}
The aim of this study was to evaluate the median survival time of fixed and removable space maintainers related to age groups, gender, and their distribution in upper and lower dental arches. The adherence of patients to a periodic recall program and the success rate of different types of space maintainers related to different arches were also evaluated. This study included 663 patients aged between 4-15 years old that were treated between the years of 1997 and 2002. The patients were categorized into four main groups: lost to follow-up, failed, successful, and censored at the end of study. Three hundred forty-five space maintainers were considered lost to follow-up, 83 were considered failed, 206 successful, and 20 censored-at-end. The overall median survival time of the appliances was 6.51 months. Median survival time was 7.25 months in the 4-6 age group, 6.35 months in the 7-12 age group, and 7.0 months in the 13+ age groups. Median survival time was 5.76 months in girls and 7.11 months in boys. Median survival time of space maintainers was 7.17 months for maxilla and 6.69 months in the mandible. Median survival time was 5.25 months for space maintainers fabricated in both arches.
\end{abstract}

Keywords: Space maintainers, life table, survival

Citation: Tulunoglu Ö, Ulusu T, Genç Y. An Evaluation of Survival of Space Maintainers: A Six-year Follow-up Study J Contemp Dent Pract 2005 February;(6)1:074-084.

(C) Seer Publishing 


\section{Introduction}

The early loss of primary molar teeth can eventually cause narrowing in the dental arch and mesialization of permanent molars resulting in crowding of teeth in the dental arch. ${ }^{1,3}$ Artun and Marstrander ${ }^{4}$ pointed out the degree of space loss shows individual dependence and is difficult to predict. However, they state that loss of space is more pronounced as follows:

1. In the maxilla than in the mandible

2. After loss of second primary molar than after loss of the first primary molar

3. More dramatic if primary molar is lost before the age of seven or eight years than if it is lost later

4. In cases with crowding of teeth than in cases with spacing

5. In retrognathic than in prognathic profiles

The most confident way to prevent the loss of space in dental arches is through the use of space maintainer appliances. The use of space maintainers, prompted by the untimely loss of a primary tooth, can prevent or reduce the severity of a developing malocclusion. ${ }^{5,6,7}$ It is best to insert a space maintainer just after the loss of teeth, as it is known the greatest amount of tooth displacement occurs within six months following the loss of teeth. ${ }^{6,7,8}$

The following factors influence the type and use of space maintainers:

1. The dentomaxillo facial growth status of the patient

2. The loss of teeth related to dental arch

3. The type of loss (unilateral or bilateral)

4. The length of the edentulous area and the number of lost teeth

5. The adaptation of the child and his parent to the treatment protocol

6. The age of the patients $\mathrm{s}^{9,10}$

Although there are several studies ${ }^{10,11,12,13}$ on different designs, indications, contraindications, and applications of space maintainers, the authors emphasize that very few studies on clinical effectiveness and longevity of space maintainers exist. $^{2,9,14}$

The aim of this study was to evaluate the median survival time of fixed and removable space main- tainers related to age groups, gender, and their distribution to upper and lower arches. Also, the compliance of patients with a periodical recall appointment program and the success rate of different space maintainers related to different arches were evaluated.

\section{Methods and Materials}

This retrospective study on evaluation of longevity of fixed and removable space maintainers was performed on a total of 663 patients aged between 4-15 years old, treated in University of Gazi Faculty of Dentistry Department Pedodontics between 1997

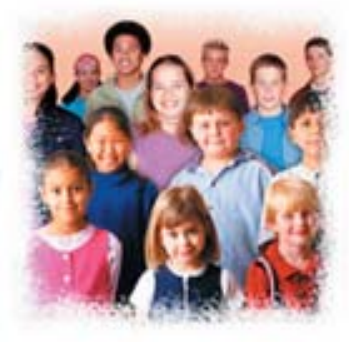
and 2002. The space maintainers were inserted under the control of two of the authors from this study using a standardized procedure.

Before space maintainer application two experienced clinicians, who conducted the study, performed the radiographic and intraoral evaluation of the subjects and analysis of study models. The patients were then referred to the Department of Orthodontics in order to verify that no orthodontic treatment would be needed. Instruction leaflets and follow-up schedules for space maintainer applications and an informed consent were given to parents of each child.

Appliance design, band selection, and impressions were made by one of the same clinicians involved in the study. All appliances were produced by the same dental technician and inserted by senior dental students under the supervision of these two authors. Fixed appliances were cemented with a glass ionomer luting cement (Logobond PD Dental D-219 Altenwalde. W. Germany). All recall appointment procedures were performed double blindly by the same clinicians. Each patient was seen for a mandatory three-month recall during which the following were performed:

- Any appliance removal with scaling

- Evaluation of the abutment teeth

- Cleaning and topical fluoride application before insertion of a new appliance 
The patients were evaluated in four main groups, respectively:

- (LF) Lost to follow-up: Patients who were non-compliant with a regular followup schedule and, consequently, space maintainers were not renewed after the first appointment.

- (F) Failed: Patients who returned after insertion of the appliance before the first appointment at the third month due to the loss or breakage of the appliance or the lack of willingness to wear the appliances. The appliances deteriorated either as a consequence of the degradation in cement breakages at the loop or soldered parts, or soft tissue lesions. Gingival submergence or decubitus and interference with the eruption sequence were classified as "failure," but the appliances in this group were renewed.

- (S) Successful: Those patients who wore their appliances and were still under observation until the eruption of permanent teeth and adhered to the recall schedule.

- (CS) Censored at the end of study: Those patients whose appliances were inserted at dates close to the ending date of the study and who were successfully wearing their appliances at that time.

The objectives of the evaluation were as follows:

1. Determination of the distribution of the subjects in the evaluation groups related to age, gender, space maintainer type, and the arch in which the appliance is inserted.

2. Assessment of the conformation of the patients to a regular follow-up schedule, the percentage of success of different space maintainers, as well as the effects of age, gender, types of appliance, and the presence in a specific dental arch.

3. The median survival (wearing) time of space maintainers related to age, gender, type of appliance, and dental arch were calculated with the life table method. The life table analysis is a method of estimating time-toevent models in the presence of censored cases. ${ }^{15}$ This method uses an actuarial approach to survival analysis that relies on partitioning the intervals and may be useful for dealing with large samples. This statistical method provides a maximum utilization of information obtained from clinical follow-up studies which involve a relationship between binary criteria and time, e.g., number of failures and successes after a certain period of observation.

Factors that might have affected the median survival time for all space maintainers were tested by using the Wilcoxon (Gehan) test.

Data were analyzed by using the SPSS (Version 10.0, SPSS Inc., Chicago, IL) statistical package. The $p$ value 0.05 and below was considered significant.

\section{Results}

The mean age of 633 patients between ages 4-15 years included in this study was $7.58 \pm 1.79$. After applying the exclusion criteria, 8 patients with 9 space maintainers were excluded. One hundred and sixty space maintainers were fabricated for the 4-6 year age group, 482 for the 7-12 age group, and 12 for the 13 and up age group. Of the 654 space maintainers, $28(4.3 \%)$ were partial dentures, $419(64.1 \%)$ were removable space maintainers, and 207 (31.6\%) were fixed space maintainers.

Distribution of the appliances related to age, gender, space maintainer type, and dental arch location. Their success and follow-up rates are presented in Table I. In the primary dentition (four to six years), 24.2 space maintainers were fitted. Of all space maintainers in the study $73.8 \%$ were fitted during the mixed dentition stage (seven to thirteen years), and in those patients older than thirteen years of age (1.9\%) during the permanent dentition stage.

Of the 633 patients, 293 were female and 340 were male. All of these space maintainers were newly constructed and fitted for the first time. Thirty patients had more than one space maintainer. The fate of all space maintainers is presented in Table II. Eighty-three were considered failures (32 fixed space maintainers, 48 removable space maintainers, and 3 removable dentures) and 206 (56 fixed space maintainers, 141 removable space maintainers, and 9 removable dentures) were successful. Twenty were considered censored and 345 were considered lost to follow-up. 
Table 1. Cross tabulation for age, gender, arch type, space maintainer type and status.

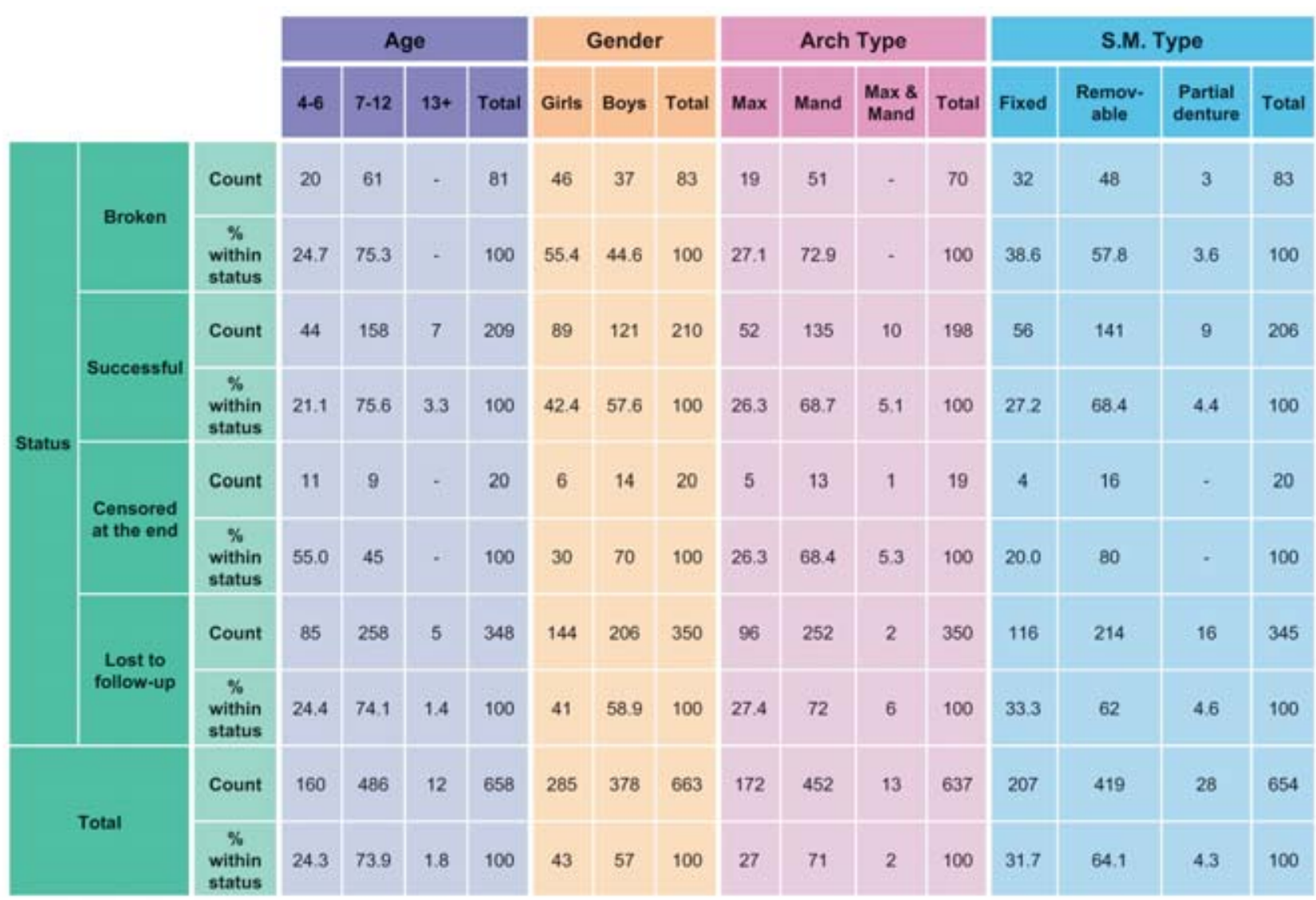

Table 2. Fate of space maintainer at the closing date.

\begin{tabular}{|c|c|c|}
\hline & Frequency & Percent \\
\hline Broken & 83 & 12.70 \\
\hline Successful & 206 & 31.5 \\
\hline Censored & 20 & 3 \\
\hline Lost to follow-up & 345 & 52.7 \\
\hline Total & 654 & 100 \\
\hline
\end{tabular}

In this study the most common failures occurred at soldered joints. The second common cause of failure was due to the free end of the loop of fixed space maintainers impinging on adjacent gingiva causing local inflammation. Although representing a relatively low percentage, the most common cause of failure in removable space maintainers was the deformation of retentive parts resulting in a decrease in retention. Another common cause of failure was fracture of the space maintainers itself. Three hundred forty-five appliances were considered as "lost to follow-up" (115 fixed space maintainers, 214 removable space maintainers, and 16 removable partial dentures). Twenty cases were considered as "Censored" (4 fixed, 16 removable).

The median survival time of a space maintainer in this present study was 6.51 months. When age groups were considered, the median survival time 
was 7.25 months in 4-6 age group, 6.35 months in 7-12 age group, and 7.0 months in $13+$ age groups. No statistically significant differences were detected between age groups. When gender was considered, the median survival time of space maintainers was 5.76 in girls and 7.11 in boys. No statistically significant differences were detected between gender groups. When dental arch location was considered, the median survival time of space maintainers was 7.17 months for the maxilla and 6.69 months in the mandible. When space maintainers were made for both arches, the median survival time was 5.25 months. No statistically significant differences were detected between arch groups. While the median survival time of fixed space maintainers was 6.16 months, this value was 6.89 for removable space maintainers and 5.40 for removable partial dentures. No significant differences were detected between space maintainer types.

The cumulative survival of space maintainers related to age, gender, and arches are shown in Figures 1, 2, 3, and 4. The general evaluation is summarized in Figure 5. Gender, age group, the arch in which the appliances were placed, and the type of the space maintainer had no significant effect on longevity $(p<0.05)$.

\section{Discussion}

Many indications for the use of space maintainers have been suggested, but very little evidence exists regarding their efficiencies. ${ }^{16}$ The major factor affecting the efficiency of space maintainers is its length of service. Therefore, the mean survival time is a valuable parameter in the indication and evaluation of space maintainers.

The life table method was reported to allow for the pooling of information from cases followed for less than the entire period of observation (i.e., cases lost to follow-up and/or inserted later), thus, calculating an average survival rate for the entire sample. ${ }^{2}$ Another advantage is the life table method allows the maximum use of data collected from clinical follow-up studies where different clinical applications are performed through a certain time period. ${ }^{15,17}$ These facts constituted the causes for preference of use of the life table method in this study.

Longitudinal studies of dental treatment have usually been carried out retrospectively, and those who attempted them will agree that the lack of record continuity presents a major difficulty. 9, 14, 16 The patients falling in the lost to follow-up group were reported to be $21 \%$ by Qudeimat and Fayle and, similarly, $19.9 \%$ by Rajab. ${ }^{14}$ In the present study the highest lost to follow-up values (52.9\%) were observed in comparison with previous studies performed by different authors. Economic constraints, the length of the treatment period, a negative attitude of parents based on the assumption crowding and misalignment of teeth could readily be treated orthodontically, or lack of adaptation of the patients to the treatment protocol causing them not to conform to recall appointments constitutes the major problems with space maintainers.

Swaine and Wright ${ }^{18}$ were the first to report use of the bonded space maintainer, but the failure rate in their study was about $30 \%$ after six months. Simonsen $^{19}$ used a commercially available perforated metallic bar and found that after one

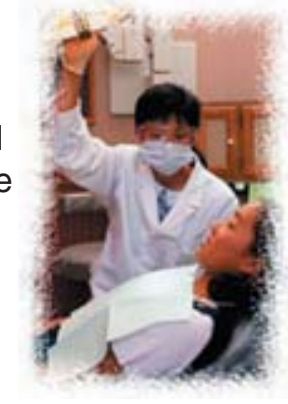
year, only one out of thirty-five appliances had debonded. Santos et al. ${ }^{8}$ evaluated clinically a fixed space maintainer, and the rate of failure reported to be $8.5 \%$. It can be considered low when compared with the results of Artun and Marstrander. ${ }^{4}$ Rajab et al. ${ }^{14}$ reported only $30.7 \%$ of the appliances experienced failure during the period of study, and the failure rate paralleled the $31.5 \%$ failure rate recorded in Baroni et al.'s study. ${ }^{2}$ These results represent a low failure rate in comparison to the failure rates of $63 \%$ and $43 \%{ }^{20}$ reported in comparable previous studies. The failure rate found in the present study $(12.7 \%)$ was lower, but it must be considered that it was performed on a relatively wider age interval and longer observation time. However, it must be noted the failure rate in the lost to follow-up group was impossible to consider, as there were no data indicating their percentage in this group. Also, the fixed space maintainer appliance types included in the previous studies ${ }^{9,14}$ showed a variety of designs including the lower lingual arch, Nance holding arch, distal shoe retainer, and band-crown/loop appliances. It has been suggested unilateral appliances were longer lasting compared to bilateral appliances. ${ }^{9}$ 


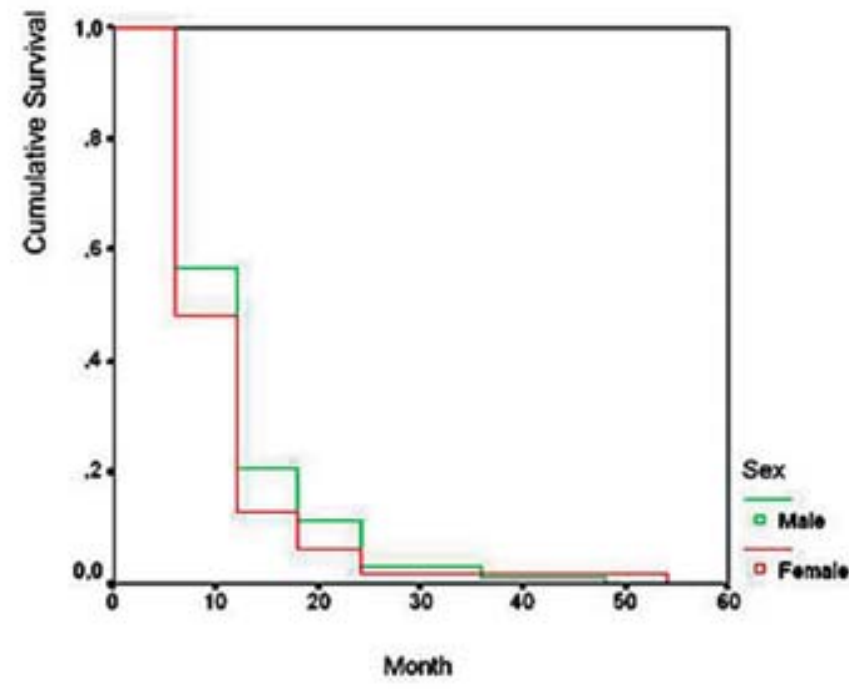

Figure 1. Cumulative survival of space maintainers related to gender.

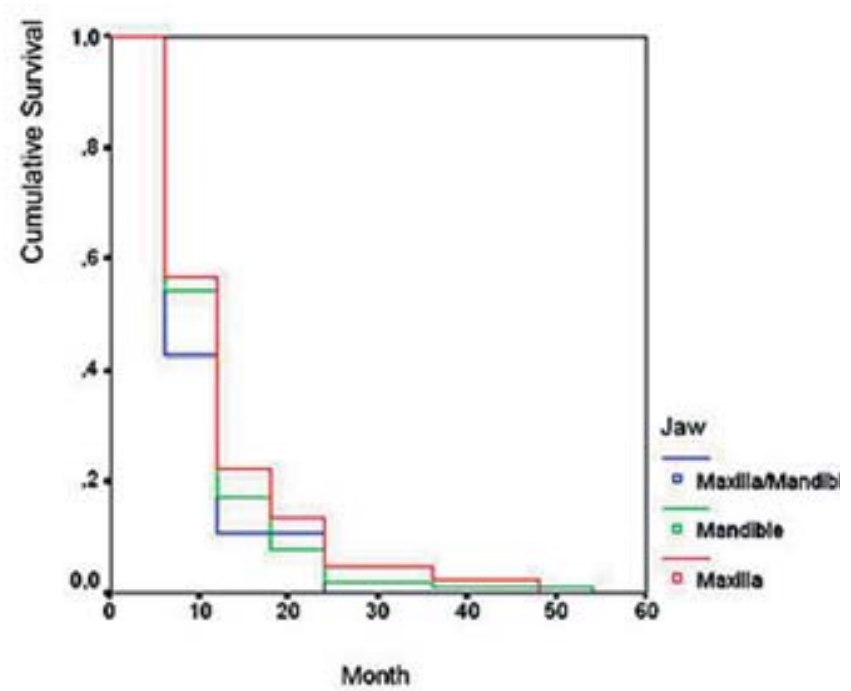

Figure 3. Cumulative survival of space maintainers related to arches.

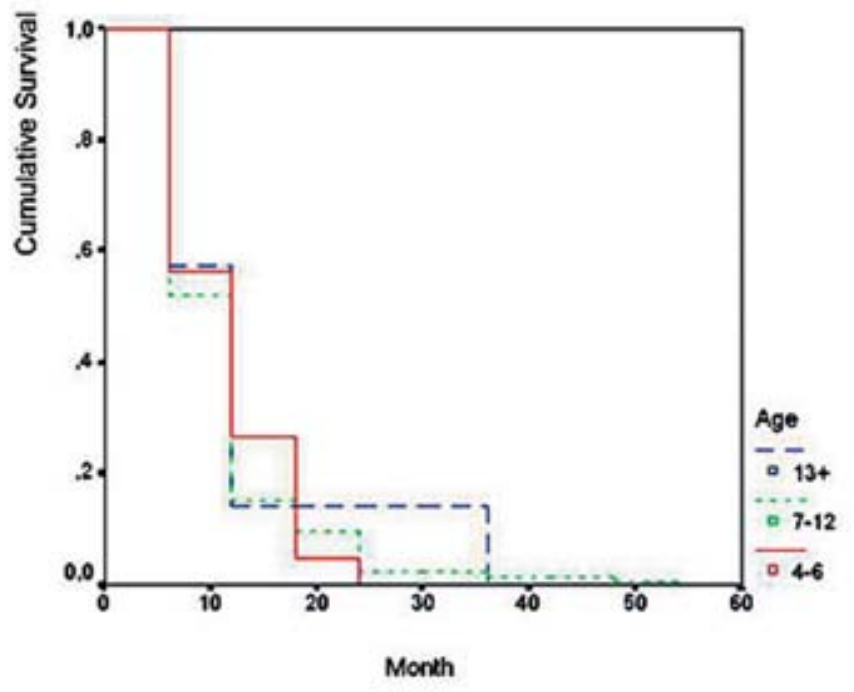

Figure 2. Cumulative survival of space maintainers related to age.

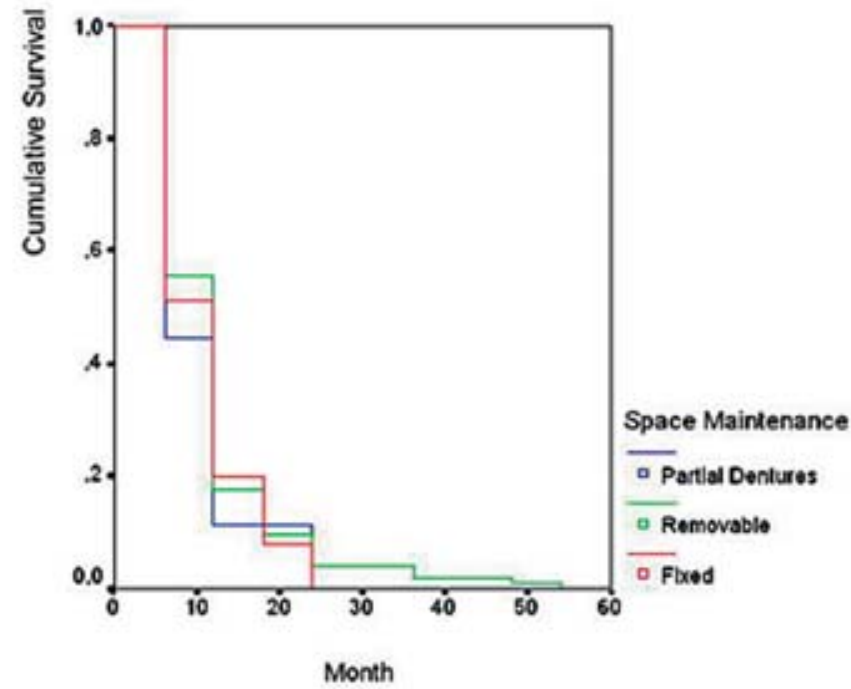

Figure 4. Cumulative survival of space maintainers related to space maintainer types.

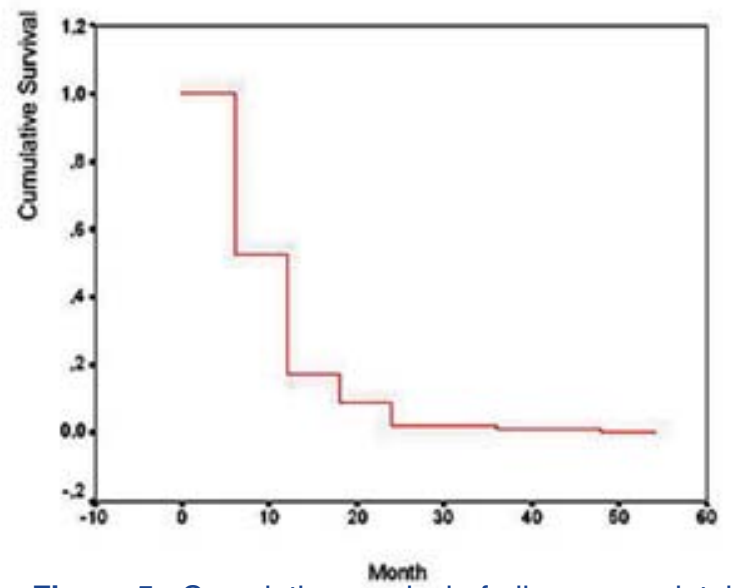

Figure 5. Cumulative survival of all space maintainers. 
The former investigators concluded the relevance of long-term mechanical stress in long-term space maintainers seemed to be more important than appliance design. ${ }^{2}$ The difficulties in properly isolating the areas related with cementation procedures and the mechanical problems occurring during mastication could present an important problem for bilateral fixed appliances, and these problems could have caused the higher failure rates in the studies that included these appliances. All these factors can be an important basis for the relatively lower failure rate observed in this present study.

The median survival time found in this study (6.51 months) was similar to that of Qudeimat et al. (7 months). However, Rajab et al. reported a higher median survival time (18 months). This survival is higher than that found in an earlier study. ${ }^{2,9}$

Qudeimat ${ }^{9}$ stated no statistically significant difference was in found their study in terms of space maintainer survival time between the different age groups. The seniority of the operator showed no statistical significance in the median survival time of the space maintainer. No statistically significant difference was found in this study in terms of space maintainer survival time between the different age groups. It has been observed the fit of space maintainers changed as a result of the growth and development process. Most of the space maintainer appliances were made during the 7-12 age range. A shorter median survival time in this study can be explained by the fact patients lose their deciduous teeth near the age of eruption of permanent teeth and, thus, the use of the space maintainer was relatively shorter.

Confirming the result of the previous investigations, gender, age, dentition, and year of placement of the appliance had no effect on survival of the space maintainers. Therefore, these findings suggest factors relating to appliance construction and cementation may be more important than other factors.
Baroni et al. ${ }^{2}$ found no significant difference between different types of space maintainers in terms of survival time. Qudeimat and Fayle ${ }^{9}$ also stated when the longevity of a fixed space maintainer was compared to removable partial-denture space maintainers, no statistically significant difference between the two different space maintainer groups was found. Fixed space maintainers were reported to last longer than removable appliances. However, the findings of the Rajab's study ${ }^{14}$, consistent with earlier investigations, found no difference between the two groups. While the results of an earlier study indicated no significant difference between the different types of space maintainers in terms of survival time, these results suggest that band and loops, Nance appliances, and removable partial dentures may have a similar probability of survival. In agreement this study found no statistically significant difference between the median survival time values of different space maintainer types.

\section{Conclusion}

The overall performance of the space maintainers can be summarized as $52.7 \%$ lost to follow-up, $12.7 \%$ failed, $3 \%$ still in use at the end of study, and $31.5 \%$ were successfully removed because they were no longer needed.

This study revealed the age, gender of the child, the arch in which the space maintainer was inserted, or the type of space maintainers included in this study did not affect the survival rate.

Space maintainers must be re-evaluated, modified, or renewed within periods of five months in order to not interfere with the growth and development processes. This will reduce the risks and in addition facilitate the wear of space maintainers. 


\section{References}

1. Rao SR. Removable partial dentures for children. In:Finn SB.(ed) Clinical Pedodontics 4th ed pp 271-286. Philadelphia, London and Toronto: W.B. Saunders Company 1973.

2. Baroni C, Franchini A, Rimondini L. Survival of different types of space maintainers. Pediatr Dent. 1994; 16:360-361.

3. Klapper BJ, Strizak-Sherwin R. Esthetic anterior space maintenance. Pediatr Dent. 1985;5: 121-123.

4. Artun J, Marstrander PB. Clinical efficiency of two different types of direct bonded space maintainers. J Dent Child. 1983; 50: 197-204.

5. Mitchell L, Mitchell DA. Oxford handbook of clinical dentistry.3 rd ed pp 128-198 Oxford: University Press, 1999.

6. Terlaje RD, Donly KJ. Treatment planning for space maintenance in the primary and mixed dentition. ASDC J Dent Child. 2001; 68: 109-114.

7. McDonald RE, Hennon DK, Avery DR. Managing space problems. In: McDonald RE, Avery DR. Eds. Dentistry for the child and adolescent. 8th pp707-744 St Louis, London, and Toronto: Mosby, 1994.

8. Santos VL, Almeida MA, Mello HSA, et. al. Direct bonded space maintainers. J Clin Pediatr Dent. 1993;17: 221-225.

9. Qudeimat MA, Fayle SA. The longevity of space maintainers: a retrospective study. Pediatr Dent. 1998; 20: 267-272.

10. Waggoner WF, Kupietzky A. Anterior esthetic fixed appliances for the preschooler: considerations and a technique for placement. Pediatr Dent. 2000; 23:147-150.

11. Liebenberg WH. Long-and short-term space maintenance following the up righting of molars: A case report. Quint Int. 1994;25: 637-640,.

12. Steffen JM, Miller JB, Johnson R. An esthetic method of anterior space maintenance. J Dent Child. 1971; 38:154-157.

13. Jasmine JR, Groper JN. Fabrication of a more durable fixed anterior esthetic appliance. J Dent Child. 1984; 51:124-127.

14. Rajab LD. Clinical performance and survival of space maintainers: Evaluation over a period of 5 years. J Dent Child. 2002; 69:156-160.

15. Thylstrup A, Rölling I. The life table method in clinical dental research. Community Dent Oral Epidemiol. 1975;3: 5-10.

16. Qudeimat MA, Fayle SA. The use of space maintainers at a UK Paediatric Dentistry department. ASDC J Dent Child. 1999; 66: 383-386.

17. Davies JA. Dental restoration longevity; a critique of the life table method of analysis. Community Dent Epidemiol. 1987;15: 202-204.

18. Swaine TJ, Wright GA, Direct bonding applied to space maintenance. J Dent Child. 1976;43: 401-5.

19. Simonsen PJ. Space maintenance utilizing acid-etch bonding. Dent Surv. 1978; 54; 27-33.

20. Hill CJ, Sorenson HW, Mink JR. Space maintenance in a child dental care program. J Am Dent Assoc. 1975; 90: 811-815. 


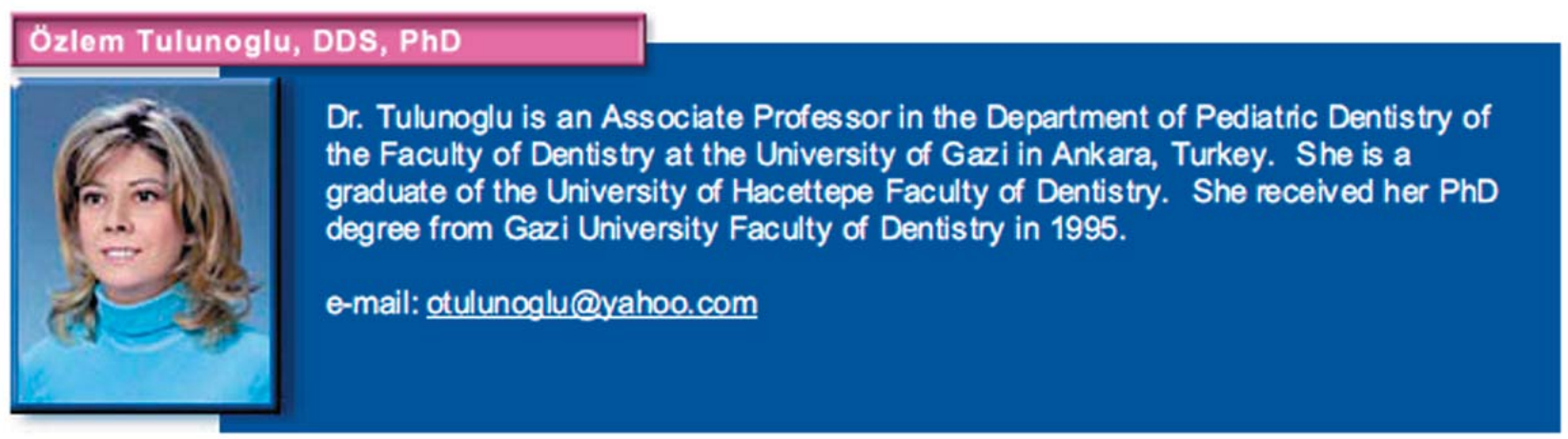

\section{Tezer Ulusu, DSD, PhD}
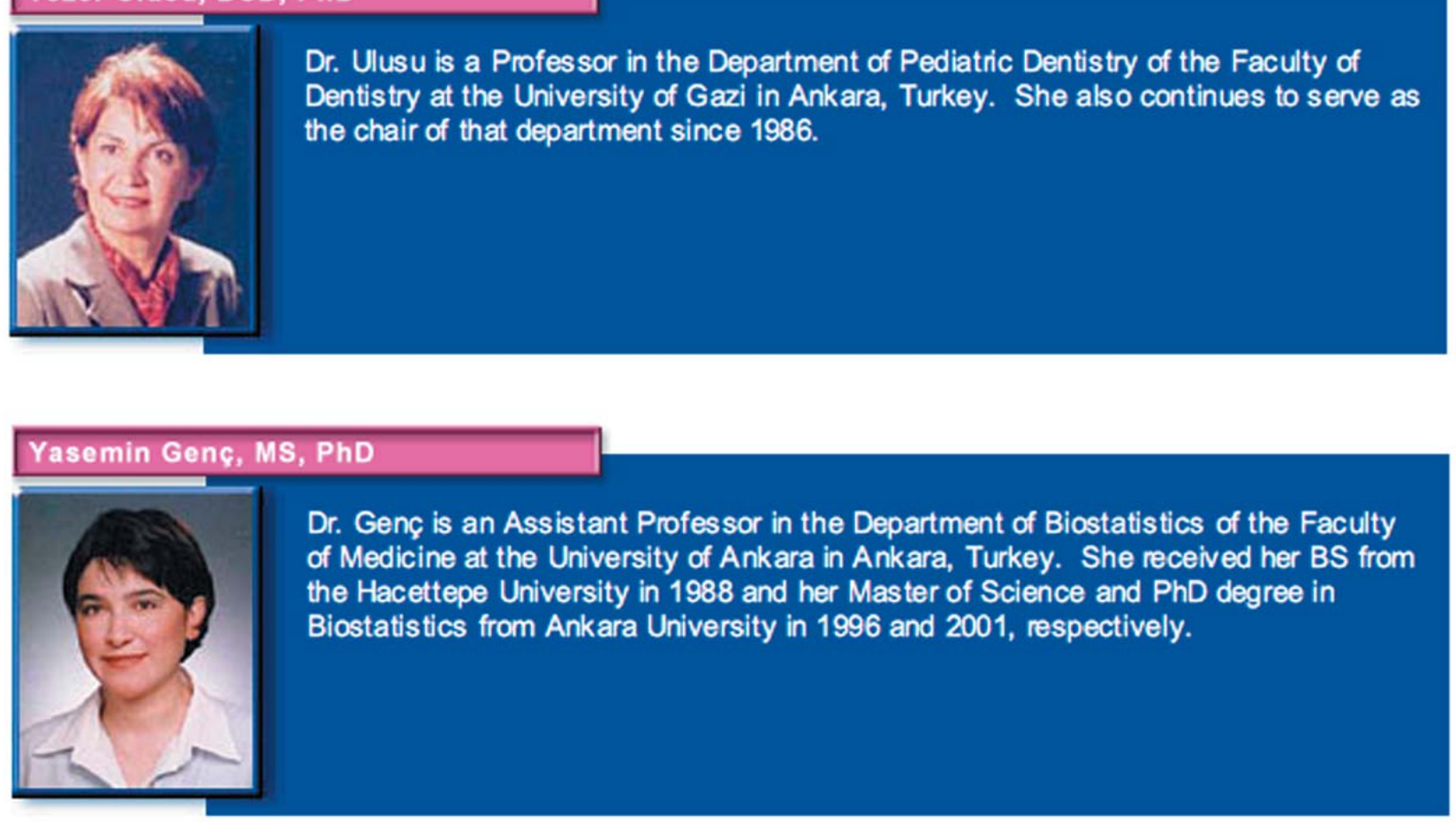